\title{
miR-425-5p Inhibits Differentiation and Proliferation in Porcine Intramuscular Preadipocytes
}

\author{
Fen-Fen Chen ${ }^{1,2,+}$, Yan Xiong ${ }^{1,+}$, Ying Peng ${ }^{1}$, Yun Gao ${ }^{1}$, Jin Qin ${ }^{1}$, Gui-Yan Chu ${ }^{1}$, \\ Wei-Jun Pang ${ }^{1}$ and Gong-She Yang ${ }^{1, *}$ \\ 1 Laboratory of Animal Fat Deposition and Muscle Development, College of Animal Science and Technology, \\ Northwest A\&F University, Yangling 712100, China; ffchen03@sina.com (F.-F.C.); \\ xiongyan0910@126.com (Y.X.); py9101@163.com (Y.P.); gy113@nwsuaf.edu.cn (Y.G.); \\ qinjin19921026@sina.com (J.Q.); yz97721@gmail.com (G.-Y.C.); pwj1226@nwsuaf.edu.cn (W.-J.P.) \\ 2 Faculty of Life Sciences, Southwest Forestry University, Kunming 650224, China \\ * Correspondence: gsyang999@hotmail.com \& gsyang@nwsuaf.edu.cn; Tel.: +86-29-8709-2430 \\ + These authors contributed equally to this work.
}

Received: 24 August 2017; Accepted: 2 October 2017; Published: 6 October 2017

\begin{abstract}
Intramuscular fat (IMF) content affects the tenderness, juiciness, and flavor of pork. An increasing number of studies are focusing on the functions of microRNAs (miRs) during porcine intramuscular preadipocyte development. Previous studies have proved that miR-425-5p was enriched in porcine skeletal muscles and played important roles in multiple physiological processes; however, its functions during intramuscular adipogenesis remain unclear. To explore the role of miR-425-5p in porcine intramuscular adipogenesis, miR-425-5p agomir and inhibitor were used to perform miR-425-5p overexpression and knockdown in intramuscular preadipocytes, respectively. Our results showed that the agomir of miR-425-5p dramatically inhibited intramuscular adipogenic differentiation and downregulated the expression levels of adipogenic marker genes PPAR $\gamma$, FABP4, and FASN, whereas its inhibitor promoted adipogenesis. Interestingly, the agomir repressed proliferation of porcine intramuscular preadipocytes by downregulation of cyclin B and cyclin E. Furthermore, we demonstrated that miR-425-5p inhibited adipogenesis via targeting and repressing the translation of KLF13. Taken together, our findings identified that miR-425-5p is a novel inhibitor of porcine intramuscular adipogenesis possibly through targeting KLF13 and subsequently downregulating PPAR $\gamma$.
\end{abstract}

Keywords: miR-425-5p; porcine intramuscular preadipocytes; proliferation; KLF13; differentiation; PPAR $\gamma$ signaling

\section{Introduction}

Intramuscular fat (IMF) content is critical for various meat quality parameters such as muscle color, firmness, and water-holding capacity [1]. Previous studies have provided the methods for isolation and in vitro culture of primary intramuscular preadipocytes, which facilitates the understanding of mechanisms of IMF deposition [2,3]. Excess adipose tissue can be the consequence of both increased fat-cell number and increased fat-cell size $[4,5]$. The number of intramuscular adipocytes present in an organism is largely determined by the intramuscular preadipocyte differentiation process $[1,6,7]$. Thus, further understanding the differentiation of pig intramuscular preadipocytes may provide valuable information in animal body development and improvement of meat quality.

MicroRNAs are a class of short and endogenously initiated non-coding RNAs that can post-transcriptionally control gene expression via either translational repression or mRNA degradation [8,9]. MiRNAs have been found to be involved in numerous physiological and pathological processes, including cell proliferation, differentiation, apoptosis, tumorigenesis, 
and neuronal development [10]. Recently, many studies have indicated that miRNAs play key roles in regulating adipogenesis [11]. In porcine adipogenesis, miR-103, miR-181a, miR-15a/b, miR-196a, miR-17, miR-21, miR-143, and miR-146a-5p promoted porcine preadipocyte differentiation [12-17], while miR-125a, miR-145, miR-191, miR-199a-5p, miR-375, and miR-429 impaired porcine preadipocyte differentiation [18-23].

Mature miR-425-5p was primarily found as a potential oncogene in cancer [24,25]. It has recently been reported to regulate cell proliferation, cell cycle, apoptosis and metastasis, immunology, and inflammation [24-29]. Recently, miR-425-5p was shown to be differently regulated during tumorigenesis and to be involved in regulating key pathways such as PI3K/AKT signaling, T cell receptor and VEGF/p38 signaling, or some of the genes which participate these processes $[25,27,28,30]$. In these biological processes, miR-425-5p functioned by targeting different mRNAs. The published targeting genes of miR-425-5p, such as insulin-like growth factor 1 (IGF-1) and mothers against decapentaplegic homolog 2 (SMAD family member 2, SMAD2), played roles in adipocyte differentiation, $[25,30]$. Here, we identified that miR-425-5p was relatively highly expressed in porcine muscle and subcutaneous adipose tissue. However, little is known about the expression and functions of miR-425-5p in porcine intramuscular adipogenesis.

In this study, we monitored the expression pattern of miR-425-5p during porcine intramuscular preadipocyte adipogenesis. Overexpression of miR-425-5p attenuates the lipid accumulation during porcine intramuscular preadipocyte differentiation, which is consistent with the block of peroxisome proliferator activated receptor $\gamma(\operatorname{PPAR} \gamma)$ signaling. Bioinformatic prediction and further experimental validation showed that Krüppel-like transcription factor 13 (KLF13) is a target of miR-425-5p in porcine adipocytes. Furthermore, we used flow cytometry, Western blot, and CCK- 8 assay to show that the overexpression of miR-425-5p levels leads to inhibiting the proliferation of porcine intramuscular preadipocytes. Taken together, our study suggests that miR-425-5p is a negative regulator for porcine intramuscular adipogenesis. Our data provides new insights in pork quality improvement.

\section{Results}

\subsection{Expression Profiles of miR-425-5p in Porcine Tissues and during Intramuscular Preadipocytes Adipogenesis}

Mature miR-425-5p sequence was highly conserved in virous mammals including pig, human, mouse, and rat (Figure 1A). To determine the tissue expression profile of miR-425-5p, total RNAs from seven tissues of adult Guanzhong Black piglets were extracted and real-time PCR (RT-PCR) was performed. Results showed (Figure 1B) that miR-425-5p was highly expressed in muscle (longissimus dorsi) and subcutaneous adipose tissue. To study the role of miR-425-5p in porcine intramuscular adipogenesis, porcine intramuscular preadipocytes were isolated. After 8 days induction, intramuscular adipocytes were fully differentiated and fulfilled with large lipid droplets (Figure 1C). Then RNA was extracted from adipogenic cells at 0, 2, 4, 6, 8, and 10 days of differentiation to detect the miR-425-5p expression pattern during intramuscular adipocyte differentiation. Our data revealed that miR-425-5p transiently increased during intramuscular adipocyte adipogenesis (Figure 1D). Taken together, these data suggested that miR-425-5p might be associated with intramuscular adipocytes adipogenesis. 
A

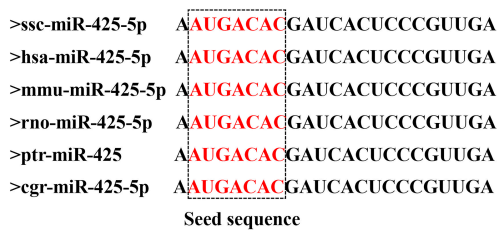

C
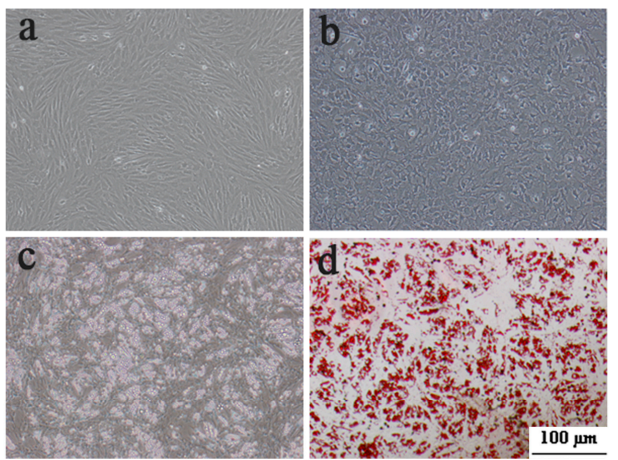

B

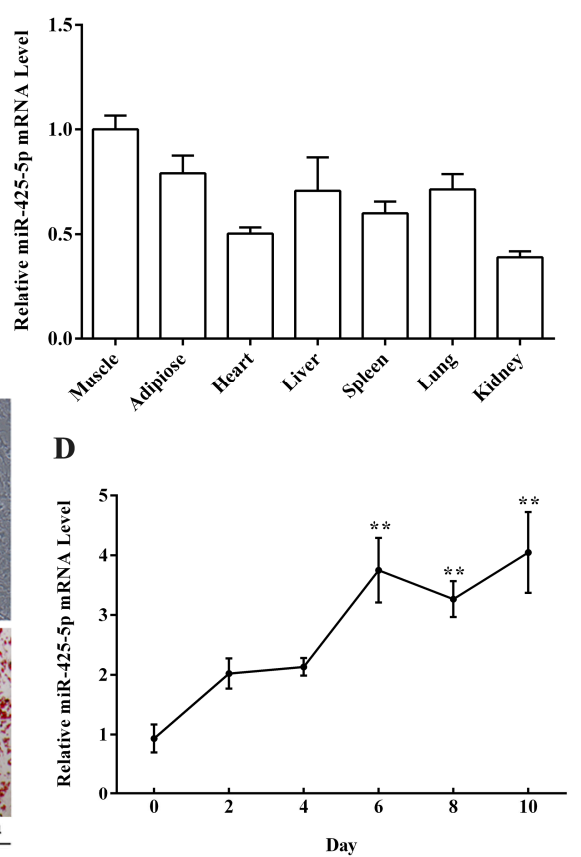

Figure 1. Expression profile of miR-425-5p in different porcine tissues and during porcine intramuscular preadipocyte differentiation. (A) Mature miR-425-5p sequence was conserved among species; (B) expression of miR-425-5p was analyzed by real-time PCR in seven different tissues of 180-day old Guanzhong Black pigs; (C) photomicrographs showing cell morphology change during porcine intramuscular preadipocyte differentiation ((a) porcine intramuscular preadipocyte before 3-isobutyl-1-methylxanthine (IBMX)-Dexamethasone (DEX)-insulin (DMI) induction; (b) porcine intramuscular preadipocyte 2 days after DMI induction; (c) porcine intramuscular preadipocyte 8 days after DMI induction; (d) porcine intramuscular preadipocyte 8 days after DMI induction staining with Oil Red O); (D) expression of miR-425-5p during porcine intramuscular preadipocyte differentiation and the expression at the day 0 as a control. U6 small nuclear RNA was used as a reference gene. Results were presented as means \pm SEM, $n=3 ;{ }^{* *} p<0.01$.

\section{2. miR-425-5p Inhibits Differentiation of Porcine Intramuscular Preadipocytes}

To identify the potential function of miR-425-5p for intramuscular adipogenesis, miR-425-5p agomir or negative control (NC) was transfected to intramuscular preadipocytes. RT-qPCR assay showed that miR-425-5p overexpressed 800-fold at the eighth day of differentiation after transfected with the agomir (Figure 2A). Gain function of miR-425-5p expression inhibited intramuscular preadipocyte differentiation indicated by reduction of both Oil Red O staining signal (Figure 2B) and triglyceride (TG) contents at day 8 of adipogenic induction $(p<0.01)$ (Figure 2C). The mRNA levels of adipogenic markers, PPAR $\gamma(p<0.01), F A B P 4(p<0.01)$, and FASN $(p<0.01)$, were significantly suppressed in miR-425-5p agomir treated cells compared with those of NC (Figure 2D). Consistently, the protein levels of PPAR $\gamma$, adipocyte fatty acid-binding protein 4 (FABP4), and fatty acid synthase (FASN) all decreased in the miR-425-5p overexpression group at day 8 of differentiation (Figure 2E).

Next, the anti-sense oligo nucleotides (inhibitor) targeting miR-425-5p or NC was transfected to porcine primary intramuscular adipocyte (the same method used for agomir transfection) to confirm the role of miR-425-5p in adipocyte differentiation. RT-qPCR showed that miR-425-5p was effectively inhibited (Figure 3A). As expected, Oil Red O staining (Figure 3B) showed an increased neutral lipid accumulation in inhibitor transfected cells. Although protein levels of FABP4 and FASN showed no difference $(p>0.05)$ (Figure 3C), this level of PPAR $\gamma$ increased significantly at day 8 of differentiation in treatment cells compared with NC $(p<0.05)$ (Figure 3C). Taken together, these data indicated that miR-425-5p inhibited porcine intramuscular preadipocyte differentiation. 
A

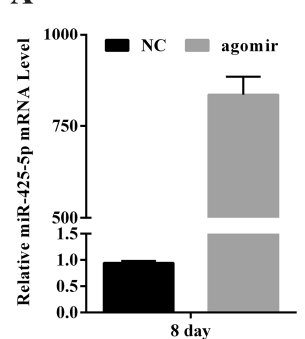

D

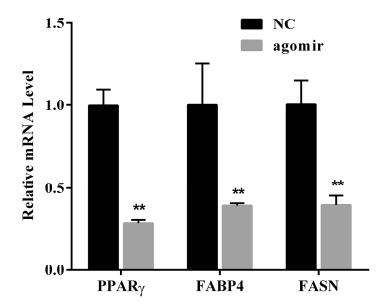

B

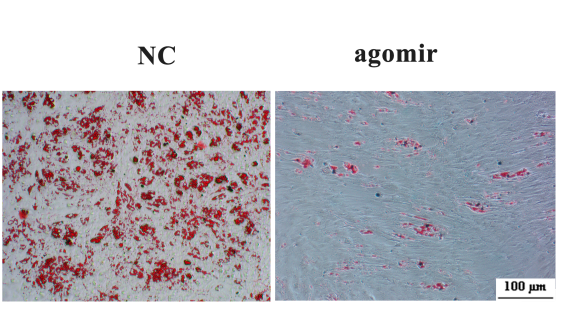

E
C

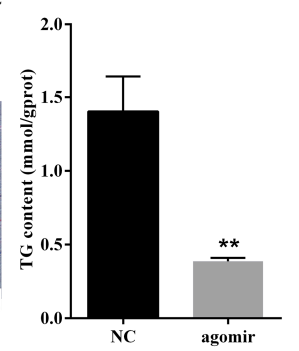

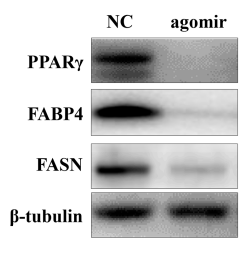

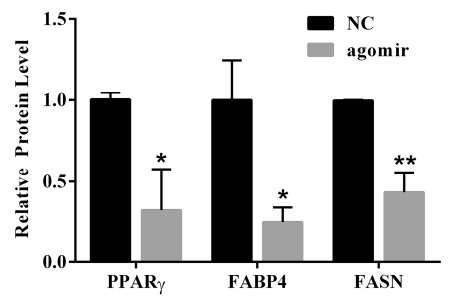

Figure 2. Overexpression of miR-425-5p inhibits porcine intramuscular adipogenesis. (A) miR-425-5p overexpression efficiency was detected by RT-qPCR; (B) the morphological changes and lipid accumulation of porcine differentiated intramuscular adipocytes observed by Oil Red $\mathrm{O}$ staining; (C) triglyceride (TG) content assay of differentiated adipocytes; (D) the relative mRNA level of adipocyte specific genes including PPAR $\gamma, F A B P 4$, and FASN; (E) protein level of PPAR $\gamma$, FABP4, and FASN genes were examined by Western blotting, $\beta$-tubulin was used as a loading control. All the cells were measure at day 8 of adipogenic differentiation. Results were presented as means \pm SEM, $n=3 ;{ }^{*} p<0.05 ;{ }^{* *} p<0.01$.

A

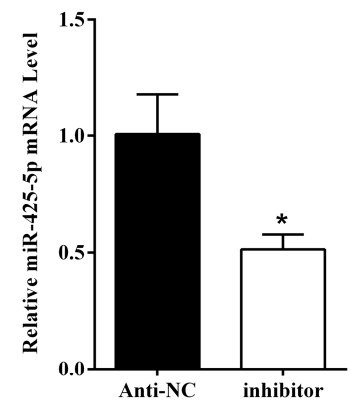

C

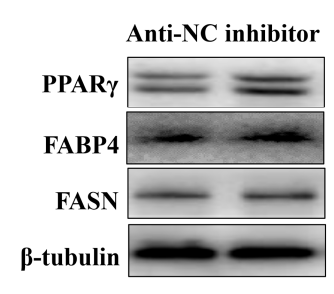

B
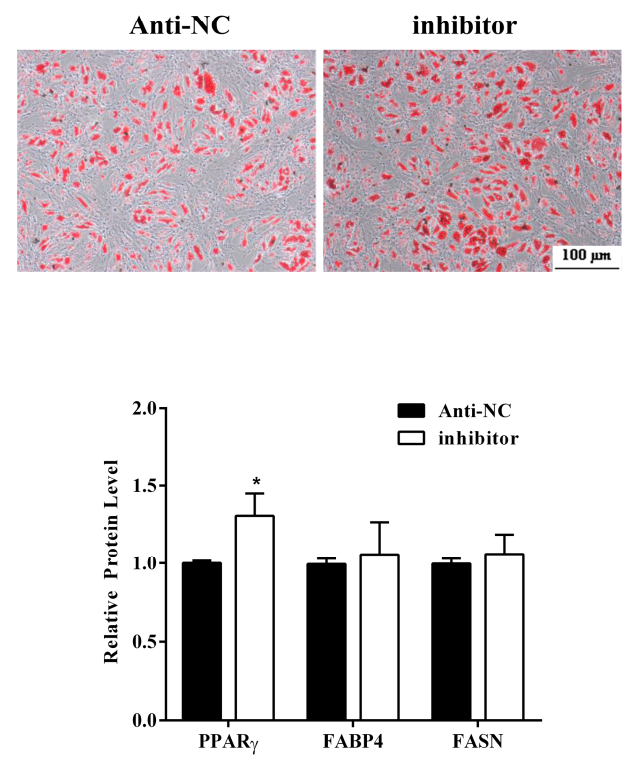

Figure 3. Inhibition of miR-425-5p promoted porcine intramuscular adipocyte differentiation. (A) The miR-425-5p expression was measured by RT-qPCR at day 8 after differentiation; (B) Oil Red O staining of intramuscular adipocytes showed antagonism of miR-425-5p increased the lipid accumulation; (C) protein expression of PPAR $\gamma$, FABP4, and FASN were determined by Western blotting, $\beta$-tubulin was used as a loading control. Results were presented as means \pm SEM, $n=3$; $* p<0.05$. 


\section{3. miR-425-5p Can Target KLF13 in Porcine Intramuscular Preadipocytes}

To reveal the underlying mechanism of miR-425-5p in porcine intramuscular preadipocyte differentiation, bioinformatic prediction software was used and found that KLF13 was a candidate target gene (Figure 4A). Therefore, the 3'UTR containing miR-425-5p targeted sites was cloned and inserted downstream of luciferase gene in the psiCHECKTM-2 reporter plasmid. Moreover, the mutated plasmid was constructed by inserting KLF13 3'UTR with mutated miR-425-5p binding site. The wild-type (psiCHECK-KLF13-UTR) or mutated (psiCHECK-KLF13-muta) plasmid was co-transfected with the miR-425-5p agomir into $293 \mathrm{~T}$ cells, respectively. After $48 \mathrm{~h}$ of transfection, the luciferase activity of the miR-425-5p group was significantly lower than that of the NC group $(p<0.05)$, and this reduction disappeared in the mutation group (Figure 4B). Further, protein levels of KLF13 were significantly decreased by miR-425-5p overexpression in porcine intramuscular adipocytes (Figure 4C). Altogether, KLF13 was a bona fide target of miR-425-5p.

A previous study proved that KLF13 is a key pro-adipogenic factor through regulating PPAR $\gamma$ transactivation during porcine adipocyte differentiation [31]. In the present study, miR-425-5p is a translational repressor of its KLF13 target gene during cell differentiation (Figure 4A,B). Overexpression of miR-425-5p in intramuscular adipocyte dramatically reduced KLF13 $(p<0.05)$ protein expression (Figure 4C), which is associated with inhibition of the expression of PPAR $\gamma(p<0.05)$ (Figure 2E). In addition, knockdown of miR-425-5p promoted the protein level of PPAR $\gamma$ (Figure 3C). Therefore, we speculated that miR-425 may indirectly regulate the expression of PPAR $\gamma$ through the targeting of KLF13.

A

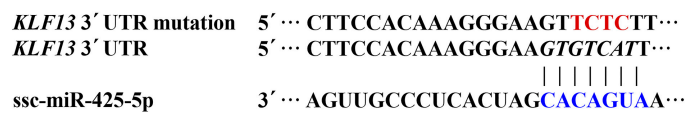

C

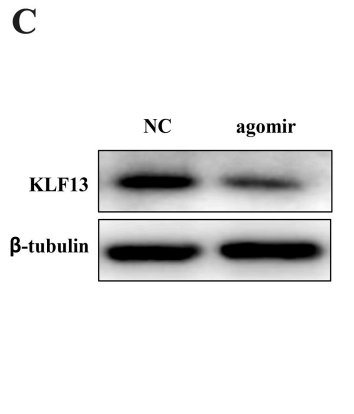

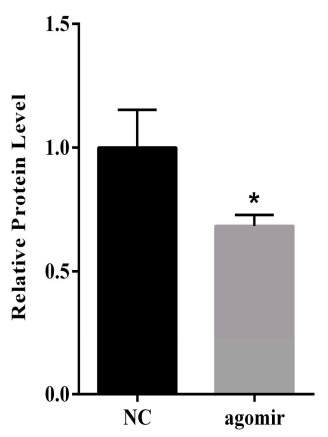

B

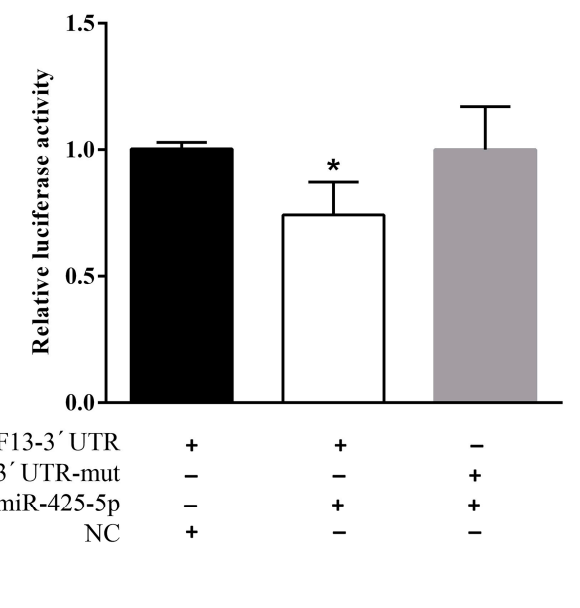

Figure 4. MiR-425-5p targets 3'UTR of KLF13. (A) Target site of miR-425-5p within porcine KLF13 mRNA $3^{\prime}$ UTR and the mutation design of its $3^{\prime} U T R$; (B) the luciferase assay of MiR-425-5p targeting $3^{\prime}$ UTR of KLF13. psiCHECK ${ }^{\mathrm{TM}}-2$ Vectors, containing either the KLF13 3'UTR or the KLF13 $3^{\prime} \mathrm{UTR}$ with a mutation in the miR-425-5p seed region, were transfected into HEK293 cells either alone or in combination with negative control (NC) or miR-425-5p agomir. Renilla luciferase activity was normalized to firefly luciferase; (C) KLF13 protein level in transfection of miR-425-5p agomir cells was detected by Western blotting at day 8 of differentiation, $\beta$-tubulin was used as a loading control. Results were presented as means $\pm \mathrm{SEM}, n=3 ; * p 0.05$.

\section{4. miR-425-5p Inhibits Porcine Intramuscular Preadipocytes Proliferation}

To elucidate whether miR-425-5p may also function in proliferating intramuscular preadipocytes, miR-425-5p agomir or NC was transfected to intramuscular preadipocytes at $40 \%$ density. After $48 \mathrm{~h}$, we analyzed the expression of miR-425-5p by RT-PCR and results showed miR-425-5p with a $6000-$ fold increase compared with the NC (Figure 5A). The mRNA expression levels of Cyclin B, a positive 
regulator of cell proliferation, was downregulated $(p<0.05)$ upon overexpression of miR-425-5p. In addition, levels of CDK4 showed no difference $(p>0.05)$ (Figure 5A). Meanwhile, compared to control, the protein levels of Cyclin B $(p<0.01)$ and Cyclin E $(p<0.05)$ were downregulated $(p<0.05)$, and levels of Cyclin D showed no difference $(p>0.05)$ (Figure 5B). After $48 \mathrm{~h}$ of transfection, we also carried out flow cytometry to detect the alterations in the cell cycle caused by miR-425-5p overexpression. The data showed that overexpressed miR-425-5p decreased the number of S-phase intramuscular preadipocytes (Figure 5C). CCK-8 assay confirmed that miR-425-5p overexpression led to decreased cell number at $48 \mathrm{~h}$ post-transfection compared to the NC group (Figure 5D). Collectively, these data indicated that miR-425-5p repressed the proliferation of porcine intramuscular preadipocytes.

A

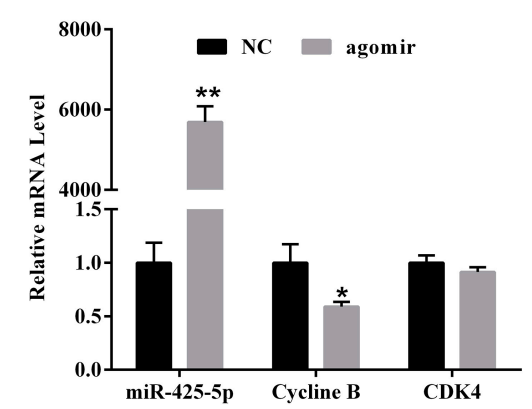

C

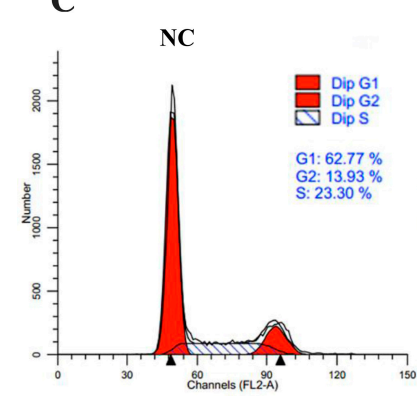

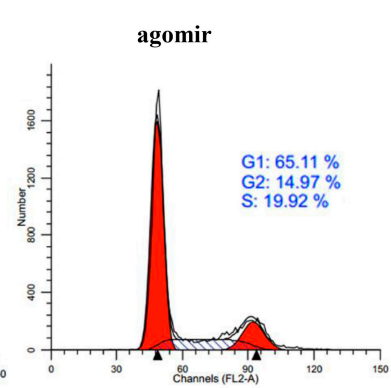

B

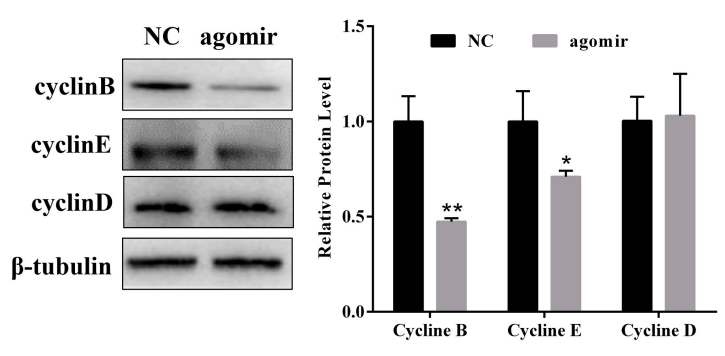

D

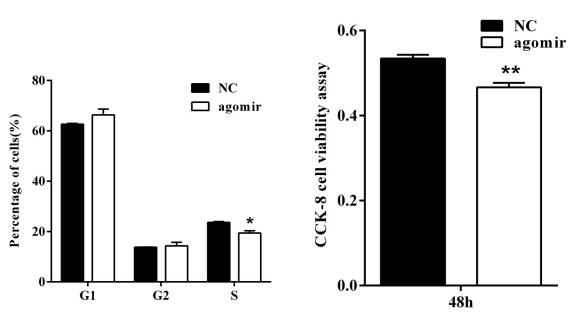

Figure 5. MiR-425-5p inhibits porcine intramuscular preadipocyte proliferation. MiR-425-5p agomir or negative control (NC) were transfected into intramuscular preadipocyte when cells reached $40 \%$ confluence and cells were harvested after $48 \mathrm{~h}$ of transfection. (A) RNA levels of miR-425-5p, Cyclin B, and CDK4 were detected by RT-qPCR; (B) the protein level of cell cycle genes, Cyclin B, Cyclin E, and Cyclin D, were analyzed by Western blotting; (C) cell-cycle analysis of intramuscular preadipocyte using flow cytometry; (D) cell proliferation was accessed by CCK-8 assay. Results were presented as mean \pm SEM, $n=3 ;{ }^{*} p<0.05$. ${ }^{* *} p<0.01$.

\section{Discussion}

Mature miR-425-5p is highly conserved in mammals, which suggests that miR-425-5p may be important for gene regulation and is thus preserved during evolution. In this study, we aimed to investigate the physiological role of miR-425-5p associated with adipogenesis in porcine intramuscular preadipocytes. We found that miR-425-5p level was increased during intramuscular preadipocyte differentiation, indicating that miR-425-5p might regulate intramuscular preadipocyte adipogenesis. We showed that the expression levels of PPAR $\gamma$, FASN, and FABP4 declined in cells transfected with miR-425-5p agomir. Consistently, blocking of miR-425-5p expression enhanced intramuscular preadipocyte differentiation. These data suggest that miR-425-5p is a negative regulator for intramuscular preadipocyte adipogenesis.

Krüppel-like factors (KLFs) are important regulators of cell differentiation, proliferation, and a number of other cellular processes [32]. In recent years, a total of 10 KLF proteins (KLF2-9, KLF13, 
and KLF15) have been proved to either promote or inhibit adipocyte differentiation [22,31]. Specifically, KLF13 has been proved to be a key pro-adipogenic factor through regulating PPAR $\gamma$ transactivation at the early stage of porcine adipocyte differentiation [31]. In this study, we showed that miR-425-5p could target KLF13, which is associated with impairing the protein expression of PPAR $\gamma$. This is further supported by the downregulated level of its downstream genes, including FABP4 and FASN [33].

Previous studies have reported that miR-425-5p displayed inconsistent effects on the proliferation of different cell types. In melanoma cells, enhancing expression of miR-425 inhibited proliferation by targeting IGF-1, which plays important roles in the PI3K/AKT signaling pathway [30]. Other studies have indicated that miR-425-5p enhanced cell proliferation via suppression of Phosphatase and tensin homolog (PTEN), SMAD2, catenin alpha 3 (CTNNA3), and cerebral cavernous malformations 3 (CCM3) expression $[25,26,29,34]$. The discrepancy of the function of miR-425-5p in different cell lines may be due to the fact that miRNA-mRNA targeting and the interplay relationship differs among tissue and cell types. Our flow cytometry and Western blot assay showed that miR-425-5p impaired the expression of cell cycle genes in intramuscular preadipocytes and significantly slowed down the cell cycle progression of these cells. For the preadipocyte adipogenic differentiation process, clonal expansion is critical for terminal adipogenic differentiation. This suggested that miR-425-5p could also restrain adipogenic differentiation by negatively regulating clonal expansion $[35,36]$.

In conclusion, our data indicated that miR-425-5p is a negative regulator of porcine intramuscular preadipocyte proliferation and differentiation by targeting KLF13. These findings provide new molecular insights into improving meat quality in the practice of animal husbandry.

\section{Materials and Methods}

\subsection{Animals}

Six Guanzhong Black piglets at 3 days were provided by the Experimental Farm of Northwest A\&F University (Yangling, China). All piglets were sacrificed by $\mathrm{CO}_{2}$ asphyxiation. The procedure was conducted in accordance with the Institutional Animal Care and Use Committee of Northwest A\&F University (14-233, 10 December 2014).

\subsection{Porcine Intramuscular Preadipocytess Isolation and Induction}

Longissimus dorsi muscles were aseptically isolated and all visible connective tissue was removed. The isolated muscle tissues were washed three times in $\mathrm{pH}$ 7.4 phosphate buffered saline (PBS) with $200 \mathrm{U} / \mathrm{mL}$ penicillin-streptomycin. The tissue was then minced to 2-3 $\mathrm{mm}$ in serum free dulbecco's modified eagle medium/F12(DMEM/F12) (Gibco BRL Co., LTD, San Francisco, CA, USA) medium with $2 \mathrm{mg} / \mathrm{mL}$ type-II collagenase (Invitrogen, Carlsbad, CA, USA), and $150 \mathrm{mg} / \mathrm{mL}$ bovine serum albumin (Sigma, St. Louis, MO, USA). The finely minced tissues were digested at $37^{\circ} \mathrm{C}$ for $120 \mathrm{~min}$ in a shaking water bath with $40 \mathrm{rpm}$ speeds. The solution added equal volume DMEM/F12 growth medium supplemented with $10 \%$ fetal bovine serum (FBS, Hyclone, Thermo scientific, Waltham, MA, USA) and $100 \mathrm{IU} / \mathrm{mL}$ penicillin-streptomycin. The digest was passed through sterile 178 and $74 \mathrm{~mm}$ steel mesh filters to isolate digested cells. Cells were rinsed with serum free DMEM/F12 medium (FBS free) and centrifuged twice at $1500 \times \mathrm{g}$ for $10 \mathrm{~min}$, and then resuspended in DMEM/F12 medium. Viable cells were counted using $0.4 \%$ trypan blue and a Countstar automatic cell counter (Inno-Alliance Biotech, Wilmington, DE, USA). Cells were resuspended in DMEM/F12 and plated at a density $6 \times 10^{5}$ per $60-\mathrm{mm}$ culture dish, and cultured in a $5 \% \mathrm{CO}_{2}$ incubator at $37^{\circ} \mathrm{C}$. Because preadipocytes attach much earlier than myoblasts, the cultures cells were rinsed with PBS three times $1 \mathrm{~h}$ after plating to remove insoluble myofibrillar proteins and other insoluble debris [2,22,37]. Cells were cultured in growth medium until they reached $80 \%$ confluence and digested with $0.05 \%$ trypsin, which contained $0.5 \mathrm{mmol} / \mathrm{L}$ EDTA, collected by centrifugation $1000 \times \mathrm{g}$ for $5 \mathrm{~min}$, then resuspended in growth medium and plated at a density of $5 \times 10^{4}$ cells $/ \mathrm{cm}$ in 6-well plate and used to induce differentiation for research. Briefly, when the cells reached confluence, the medium was changed with induction medium, 
which is the DMEM/F12 supplement with $10 \%$ FBS, $100 \mathrm{U} / \mathrm{mL}$ penicillin-streptomycin, $0.5 \mathrm{mM} \mathrm{IBMX,}$ $1 \mathrm{nM}$ DEX, and $5 \mathrm{ng} / \mathrm{mL}$ insulin (IBMX, DEX and insulin were purchased from Sigma). After 2 days, the medium was changed with differentiation medium, including DMEM/F12 supplement with 10\% FBS, $5 \mathrm{ng} / \mathrm{mL}$ insulin, and $100 \mathrm{U} / \mathrm{mL}$ penicillin-streptomycin, then changed every 2 days until day 8 . At this stage, the cells were differentiated and used for subsequent experiments.

\subsection{Transfection of miRNA Agomir and Inhibitor}

Porcine intramuscular preadipocytes were seeded in 12-well or 6-well plates, and $50 \mathrm{nM}$ miR-425-5p agomir or negative control (NC) (Genepharma, Shanghai, China) was transfected into cells of $40 \%$ density using X-tremeGENE siRNA Transfection Reagent (No.04476115001, Roche Diagnostics $\mathrm{GmbH}$, Mannheim, Germany) and Opti-MEM (Gibco BRL Co., LTD) culture medium according to the manufacturers' protocol. After $48 \mathrm{~h}$, the culture medium was changed to fresh medium in order to study the proliferation of intramuscular preadipocytes. Cells were harvested $48 \mathrm{~h}$ after transfection. When transfected with miR-425-5p inhibitor, the protocol was the same as with agomir, but the final concentration of miR-425-5p inhibitor was $100 \mathrm{nM}$, according to the introductions. Nevertheless, for adipogenic differentiation, cells were transfected when density of intramuscular preadipocytes reached $70 \%$. When cells grew to confluence after transfection, adipogenic differentiation was initiated by switching to differentiation medium.

\subsection{RNA Extraction and RT-PCR}

Total RNA was extracted from the cells using TRIzol reagent (Takara, Kyoto, Japan). cDNA was synthesized and RT-qPCR reactions were performed in triplicate using the SYBR green kit (Takara) with a Bio-Rad iQ ${ }^{\mathrm{TM}} 5$ system. microRNA assay was detected with stem-loop primers purchased from RiboBio. U6 small nucleolar RNA (Guangzhou RiboBio Co., LTD, Guangzhou, China) was used for the normalization. The primer sequences used for genes studied are listed in Table 1. The final relative expression fold differences, with gene expression $\mathrm{NC}$ as a control, were calculated as $2^{-\Delta \Delta C t}$ for each gene.

Table 1. Primer sequences used in this study.

\begin{tabular}{ccccc}
\hline Gene & Accession Number & Orientation & Primer Sequences $\left(\mathbf{5}^{\prime} \mathbf{- 3}^{\prime} \mathbf{)}\right.$ & Production Length (bp) \\
\hline PPAR $\gamma$ & NM_214379 & $\begin{array}{c}\text { Forward } \\
\text { Reverse }\end{array}$ & $\begin{array}{c}\text { AGGACTACCAAAGTGCCATCAAA } \\
\text { GAGGCTTTACCCCACAGACAC }\end{array}$ & 142 \\
\hline FABP4 & HM_453202 & $\begin{array}{c}\text { Forward } \\
\text { Reverse }\end{array}$ & $\begin{array}{c}\text { GAGCACCATAACCTTAGATGGA } \\
\text { AAATTCTGGTAGCCGTGACA }\end{array}$ & \multirow{2}{*}{121} \\
\hline \multirow{2}{*}{ FASN } & EF589048.1 & $\begin{array}{c}\text { Forward } \\
\text { Reverse }\end{array}$ & $\begin{array}{c}\text { AGCCTAACTCCTCGCTGCAAT } \\
\text { TCCTTGGAACCGTCTGTGTTC }\end{array}$ & \multirow{2}{*}{196} \\
\hline Cyclin B & NM_001170768 & $\begin{array}{c}\text { Forward } \\
\text { Reverse }\end{array}$ & $\begin{array}{c}\text { AATCCCTTCTTGTGGTTA } \\
\text { CTTAGATGGGCATACTTG }\end{array}$ & \multirow{2}{*}{104} \\
\hline CDK4 & NM_001123097 & $\begin{array}{c}\text { Forward } \\
\text { Reverse }\end{array}$ & $\begin{array}{c}\text { ATCAGCACGGTTCGTGAAGT } \\
\text { GCTCAAACACCAGGGTCACT }\end{array}$ & 133 \\
\hline GAPDH & KJ786424 & $\begin{array}{c}\text { Forward } \\
\text { Reverse }\end{array}$ & $\begin{array}{c}\text { AGGTCGGAGTGAACGGATTTG } \\
\text { ACCATGTAGTGGAGGTCAATGAAG }\end{array}$ & 118 \\
\hline
\end{tabular}

\subsection{Western Blotting}

As previously described [22], cells were washed twice with PBS and solubilized with radio immunoprecipitation assay (RIPA) buffer (Vazyme Biotec Co., LTD, Nanjing, China) supplemented with protease inhibitor (Sigma). Lysates were quantitated with bicinchoninic acid (BCA) kit (Vazyme Biotec Co., LTD); equivalent amounts of protein were subjected to electrophoresis, and transferred onto PVDF membranes (Millipore, Billerica, MA, USA). Blocking with 5\% dried skimmed milk in TBST (Tris Buffered Saline and $0.1 \%$ Tween 20) for $2 \mathrm{~h}$ at room temperature, the membranes were incubated with different primary antibodies, including KLF13 (\#41724, SAB, Maryland, MD, USA), FASN (sc-20140 
AC, Santa Cruz Biotechnology, Inc., Santa Cruz, CA, USA), FABP4 (sc-18661, Santa Cruz Biotechnology, Inc.), PPAR $\gamma$ (\#2435, CST, Cell Signaling Technology, Inc., Danvers, MA, USA), and $\beta$-tubulin (KM9003, Sungene, Tianjin, China), at $4{ }^{\circ} \mathrm{C}$ overnight. After washing, the membrane was incubated with HRP-conjugated secondary antibodies for $1 \mathrm{~h}$ at room temperature and developed with ChemiDoc ${ }^{\mathrm{TM}}$ XRS + Chem iluminescence detection system (Bio-Rad, Hercules, CA, USA). Image Lab5.2 was used for densitometric analysis of the expressed protein bands.

\subsection{Oil Red O Staining}

The extent of differentiation was determined by the amount of lipid accumulation by Oil Red O (ORO) staining, as described previously [38]. Cells were observed and photographed by TE2000-S microscope (Nikon, Tokyo, Japan).

\subsection{Measurement TG}

TG contents were detected on day 8 of differentiation. Cells were digested by $0.25 \%$ trypsin and then homogenized by ultrasonic cracking (Sonics Vcx105, Newtown, CT, USA). Lysates were measured using TG test kit (Nanjing Jiancheng Bioengineering Institute, Nanjing, China). TG content analysis was conducted according to the manufacturer's protocol.

\subsection{Luciferase Reporter Assay}

The 3'-UTRs of porcine KLF13 containing miR-425-5p targeted sites were cloned from porcine adipocytes cDNA using primers tagged with XhoI and NotI (Takar) cutting sites. The wild-type or mutated $3^{\prime}$-UTR fragment was cloned into psiCHECKTM-2 Vector (Promega, Madison, WI, USA) at the $3^{\prime}$-end of the Renilla gene. The structured 3'-UTR dual-luciferase vectors/the 3'-UTR point mutations at positions 2-6 of the KLF13 seed region dual-luciferase vectors and miR-425-5p agomir/NC were co-transfected into porcine intramuscular preadipocyte using X-tremeGENE HP DNA Transfection Reagent. Cells were harvested at $48 \mathrm{~h}$ post-transfection and assayed based on the manufacturer's instructions (Promega).

\subsection{Flow Cytometry}

Porcine intramuscular preadipocytes being the stable phase of cell growth, cells were seeded in 6-well culture plates at a density of $1.6 \times 10^{6}$ cells per well. After $24 \mathrm{~h}$, cells were transfected with miR-425-5p agomir/NC by X-tremeGENE HP siRNA Transfection Reagent (Roche), and cells were washed three times with PBS, harvested at $48 \mathrm{~h}$ post-transfection, and then stained with DNA staining solution (CCS01; Multi Sciences, Hangzhou, China), and left to incubate for 30 min. Finally, a flow cytometry instrument (Becton Dickinson, Franklin Lakes, NJ, USA) was used to analyze the proliferation phase of the cells.

\subsection{Cell Vitality Analysis}

Porcine intramuscular preadipocytes were seeded in 96-well plates in the growth medium with a density of $2 \times 10^{3}$ cells per well. After transfection of agomir/NC for $24 \mathrm{~h}$, the medium was removed and $90 \mu \mathrm{L}$ or fresh medium mix with $10 \mu \mathrm{L}$ CCK-8 reagents (Vazyme Biotec Co., LTD) were added to each well. After incubation for $2 \mathrm{~h}$ at $37^{\circ} \mathrm{C}$, the 96 -well culture plates were agitated for $1 \mathrm{~min}$ and the absorbance at $450 \mathrm{~nm}$ for each well was detected using a PerkinElmer VICTOR X5 (PerkinElmer lnc, Waltham, MA, USA).

\subsection{Statistical Analysis}

All experiments were carried out at least three times. GraphPad Prism 6.0 was utilized to graph the results. Data were analyzed using SPSS 17.0 software (SPSS science, Chicago, IL, USA) and evaluated 
for differences between groups by unpaired Student's $t$-test. Data are presented as means \pm SEM. A value of $p<0.05\left(^{*}\right)$ was considered to be statistically significant.

Acknowledgments: This work was supported by the National Key Basic Research Programs of China (2012CB124705), the National Nature Science Foundation of China (31572366 and U1201213), and the National Nature Science Foundation of Shanxi Province (2015JM3096).

Author Contributions: Fen-Fen Chen, Yan Xiong, and Gong-She Yang conceived and designed the experiments; Fen-Fen Chen, Ying Peng, Jin Qin, and Gui-Yan Chu performed the experiments; Fen-fen Chen, Yan Xiong, and Ying Peng analyzed the data; Fen-Fen Chen, Yan Xiong, Ying Peng, Wei-Jun Pang, and Gong-She Yang wrote the paper; Yan Xiong and Fen-Fen Chen revised this paper and all authors reviewed the final manuscript.

Conflicts of Interest: The authors declare no conflict of interest.

\begin{tabular}{ll}
\multicolumn{2}{l}{ Abbreviations } \\
PPAR $\gamma$ & Peroxisome proliferator activated receptor $\gamma$ \\
FABP4 & Adipocyte fatty acid-binding protein 4 \\
FASN & Fatty acid synthase \\
KLF13 & Krüppel-like transcription factor 13 \\
Cyclin E & Cell cycle protein E \\
Cyclin B & Cell cycle protein B \\
Cyclin D & Cell cycle protein D \\
CDK4 & Cyclin-dependent kinases 4 \\
ssc & Susscrofa \\
hsa & Homo sapiens \\
mmu & Musmusculus \\
rno & Rattusnorvegicus \\
ptr & Pan troglodytes \\
cgr & Cricetulusgriseus
\end{tabular}

\section{References}

1. Hocquette, J.F.; Gondret, F.; Baeza, E.; Medale, F.; Jurie, C.; Pethick, D.W. Intramuscular fat content in meat-producing animals: Development, genetic and nutritional control, and identification of putative markers. Anim. Int. J. Anim. Biosci. 2010, 4, 303-319. [CrossRef] [PubMed]

2. Zhao, C.Z.; Chen, X.C.; Wu, W.J.; Wang, W.S.; Pang, W.J.; Yang, G.S. Mat2b promotes adipogenesis by modulating same levels and activating akt/erk pathway during porcine intramuscular preadipocyte differentiation. Exp. Cell Res. 2016, 344, 11-21. [CrossRef] [PubMed]

3. Sun, W.X.; Dodson, M.V.; Jiang, Z.H.; Yu, S.G.; Chu, W.W.; Chen, J. Myostatin inhibits porcine intramuscular preadipocyte differentiation in vitro. Domest. Anim. Endocrinol. 2016, 55, 25-31. [CrossRef] [PubMed]

4. Berry, R.; Jeffery, E.; Rodeheffer, M.S. Weighing in on adipocyte precursors. Cell Metab. 2014. [CrossRef] [PubMed]

5. Poulos, S.P.; Dodson, M.V.; Hausman, G.J. Cell line models for differentiation: Preadipocytes and adipocytes. Exp. Biol. Med. 2010, 235, 1185-1193. [CrossRef] [PubMed]

6. Chen, X.L.; Luo, Y.L.; Huang, Z.Q.; Jia, G.; Liu, G.M.; Zhao, H. Role of phosphotyrosine interaction domain containing 1 in porcine intramuscular preadipocyte proliferation and differentiation. Anim. Biotechnol. 2016, 27, 287-294. [CrossRef] [PubMed]

7. Dodson, M.V.; Allen, R.E.; Du, M.; Bergen, W.G.; Velleman, S.G.; Poulos, S.P.; Fernyhough-Culver, M.; Wheeler, M.B.; Duckett, S.K.; Young, M.R.; et al. Invited review: Evolution of meat animal growth research during the past 50 years: Adipose and muscle stem cells. J. Anim. Sci. 2015, 93, 457-481. [CrossRef] [PubMed]

8. Bartel, D.P. Micrornas: Genomics, biogenesis, mechanism, and function. Cell 2004, 116, 281-297. [CrossRef]

9. He, L.; Hannon, G.J. Micrornas: Small RNAs with a big role in gene regulation. Nat. Rev. Genet. 2004, 5, 522-531. [CrossRef] [PubMed]

10. Tufekci, K.U.; Meuwissen, R.L.; Genc, S. The role of micrornas in biological processes. Methods Mol. Biol. 2014, 1107, 15-31. [PubMed] 
11. Wang, Z.; Qiao, Y.; Zhang, J.; Shi, W.; Zhang, J. Genome wide identification of micrornas involved in fatty acid and lipid metabolism of brassica napus by small RNA and degradome sequencing. Gene 2017, 619, 61-70. [CrossRef] [PubMed]

12. Li, M.; Qiu, Y.; Liu, S.; Dong, P.; Ning, X.; Li, Y.; Yang, G.; Sun, S. Over-expressed miR-103 promotes porcine adipocyte differentiation. Chin. J. Biotechnol. 2012, 28, 927-936.

13. Li, H.; Chen, X.; Guan, L.; Qi, Q.; Shu, G.; Jiang, Q.; Yuan, L.; Xi, Q.; Zhang, Y. MiRNA-181a regulates adipogenesis by targeting tumor necrosis factor- $\alpha$ (TNF- $\alpha$ ) in the porcine model. PLoS ONE 2013, 8, e71568. [CrossRef] [PubMed]

14. Dong, P.; Mai, Y.; Zhang, Z.; Mi, L.; Wu, G.; Chu, G.; Yang, G.; Sun, S. miR-15a/b promote adipogenesis in porcine pre-adipocyte via repressing foxo1. Acta Biochim. Biophys. Sin. 2014, 46, 565-571. [CrossRef] [PubMed]

15. An, X.; Ma, K.; Zhang, Z.; Zhao, T.; Zhang, X.; Tang, B.; Li, Z. miR-17, miR-21 and miR-143 enhance adipogenic differentiation from porcine bone marrow-derived mesenchymal stem cells. DNA Cell Biol. 2016, 35, 410-416. [CrossRef] [PubMed]

16. Ning, X.; Liu, S.; Qiu, Y.; Li, G.; Li, Y.; Li, M.; Yang, G. Expression profiles and biological roles of miR-196a in swine. Genes 2016, 7, 5. [CrossRef] [PubMed]

17. Wu, D.; Xi, Q.Y.; Cheng, X.; Dong, T.; Zhu, X.T.; Shu, G.; Wang, L.N.; Jiang, Q.Y.; Zhang, Y.L. miR-146a-5p inhibits TNF- $\alpha$-induced adipogenesis via targeting insulin receptor in primary porcine adipocytes. J. Lipid Res. 2016, 57, 1360-1372. [CrossRef] [PubMed]

18. Guo, Y.; Chen, Y.; Zhang, Y.; Zhang, Y.; Chen, L.; Mo, D. Up-regulated miR-145 expression inhibits porcine preadipocytes differentiation by targeting IRS1. Int. J. Biol. Sci. 2012, 8, 1408-1417. [CrossRef] [PubMed]

19. Ji, H.L.; Song, C.C.; Li, Y.F.; He, J.J.; Li, Y.L.; Zheng, X.L.; Yang, G.S. miR-125a inhibits porcine preadipocytes differentiation by targeting erralpha. Mol. Cell. Biochem. 2014, 395, 155-165. [CrossRef] [PubMed]

20. Shi, X.E.; Li, Y.F.; Jia, L.; Ji, H.L.; Song, Z.Y.; Cheng, J.; Wu, G.F.; Song, C.C.; Zhang, Q.L.; Zhu, J.Y.; et al. MicroRNA-199a-5p affects porcine preadipocyte proliferation and differentiation. Int. J. Mol. Sci. 2014, 15, 8526-8538. [CrossRef] [PubMed]

21. Liu, S.; Sun, G.; Yuan, B.; Zhang, L.; Gao, Y.; Jiang, H.; Dai, L.; Zhang, J. miR-375 negatively regulates porcine preadipocyte differentiation by targeting BMPR2. FEBS Lett. 2016, 590, 1417-1427. [CrossRef] [PubMed]

22. Peng, Y.; Chen, F.F.; Ge, J.; Zhu, J.Y.; Shi, X.E.; Li, X.; Yu, T.Y.; Chu, G.Y.; Yang, G.S. miR-429 inhibits differentiation and promotes proliferation in porcine preadipocytes. Int. J. Mol. Sci. 2016, 17, 27. [CrossRef] [PubMed]

23. Ji, S.; Li, W.; Bao, L.; Han, P.; Yang, W.; Ma, L.; Meng, F.; Cao, B. Pu.1 promotes miR-191 to inhibit adipogenesis in 3T3-L1 preadipocytes. Biochem. Biophys. Res. Commun. 2014, 451, 329-333. [CrossRef] [PubMed]

24. Liu, J.; Li, T.; Zhang, N.; Yang, X.; Wang, Z.; Ma, J.; Gu, X.; Fan, Y.; Cai, D. miR-425 up-regulation induced by interleukin-1beta promotes the proliferation of gastric cancer cell ags. Zhonghua Yi Xue Za Zhi 2014, 94, 1889-1893. (In Chinese) [PubMed]

25. Liu, L.; Zhao, Z.; Zhou, W.; Fan, X.; Zhan, Q.; Song, Y. Enhanced expression of miR-425 promotes esophageal squamous cell carcinoma tumorigenesis by targeting SMAD2. J. Genet. Genom. 2015, 42, 601-611. [CrossRef] [PubMed]

26. Gao, Y.; Yin, Y.; Xing, X.; Zhao, Z.; Lu, Y.; Sun, Y.; Zhuang, Z.; Wang, M.; Ji, W.; He, Y. Arsenic-induced anti-angiogenesis via miR-425-5p-regulated CCM3. Toxicol. Lett. 2016, 254, 22-31. [CrossRef] [PubMed]

27. Chen, X.; Dong, H.; Liu, S.; Yu, L.; Yan, D.; Yao, X.; Sun, W.; Han, D.; Gao, G. Long noncoding RNA mhencr promotes melanoma progression via regulating miR-425/489-mediated pi3k-akt pathway. Am. J. Transl. Res. 2017, 9, 90-102. [PubMed]

28. Nakagawa, R.; Muroyama, R.; Saeki, C.; Goto, K.; Kaise, Y.; Koike, K.; Nakano, M.; Matsubara, Y.; Takano, K.; Ito, S.; et al. miR-425 regulates inflammatory cytokine production in CD4+ T cells via n-Ras upregulation in primary biliary cholangitis. J. Hepatol. 2017, 66, 1223-1230. [CrossRef] [PubMed]

29. Zhang, Z.; Wen, M.; Guo, J.; Shi, J.; Wang, Z.; Tan, B.; Zhang, G.; Zheng, X.; Zhang, A. Clinical value of miR-425-5p detection and its association with cell proliferation and apoptosis of gastric cancer. Pathol. Res. Pract. 2017, 213, 929-937. [CrossRef] [PubMed]

30. Liu, P.; Hu, Y.; Ma, L.; Du, M.; Xia, L.; Hu, Z. MiR-425 inhibits melanoma metastasis through repression of pi3k-akt pathway by targeting IGF-1. Biomed. Pharmacother. 2015, 75, 51-57. [CrossRef] [PubMed] 
31. Jiang, S.; Wei, H.; Song, T.; Yang, Y.; Zhang, F.; Zhou, Y.; Peng, J.; Jiang, S. Klf13 promotes porcine adipocyte differentiation through ppargamma activation. Cell Biosci. 2015, 5, 28. [CrossRef] [PubMed]

32. Pearson, R.; Fleetwood, J.; Eaton, S.; Crossley, M.; Bao, S. Kruppel-like transcription factors: A functional family. Int. J. Biochem. Cell Biol. 2008, 40, 1996-2001. [CrossRef] [PubMed]

33. Rosen, E.D.; Spiegelman, B.M. What we talk about when we talk about fat. Cell 2014, 156, 20-44. [CrossRef] [PubMed]

34. He, B.; Li, T.; Guan, L.; Liu, F.E.; Chen, X.M.; Zhao, J.; Lin, S.; Liu, Z.Z.; Zhang, H.Q. Ctnna3 is a tumor suppressor in hepatocellular carcinomas and is inhibited by miR-425. Oncotarget 2016, 7, 8078-8089. [CrossRef] [PubMed]

35. Rosen, E.D.; Spiegelman, B.M. Molecular regulation of adipogenesis. Annu. Rev. Cell Dev. Biol. 2000, 16, 145-171. [CrossRef] [PubMed]

36. Feve, B. Adipogenesis: Cellular and molecular aspects. Best Pract. Res. Clin. Endocrinol. Metab. 2005, 19, 483-499. [CrossRef] [PubMed]

37. Wang, G.Q.; Zhu, L.; Ma, M.L.; Chen, X.C.; Gao, Y.; Yu, T.Y.; Yang, G.S.; Pang, W.J. Mulberry 1-deoxynojirimycin inhibits adipogenesis by repression of the Erk/ppargamma signaling pathway in porcine intramuscular adipocytes. J. Agric. Food Chem. 2015, 63, 6212-6220. [CrossRef] [PubMed]

38. Pang, W.; Wang, Y.; Wei, N.; Xu, R.; Xiong, Y.; Wang, P.; Shen, Q.; Yang, G. Sirt1 inhibits AKT2-mediated porcine adipogenesis potentially by direct protein-protein interaction. PLoS ONE 2013, 8, e71576. [CrossRef] [PubMed]

(C) 2017 by the authors. Licensee MDPI, Basel, Switzerland. This article is an open access article distributed under the terms and conditions of the Creative Commons Attribution (CC BY) license (http:/ / creativecommons.org/licenses/by/4.0/). 